\title{
Longitudinal Associations Between IIIness Perceptions and Glycemic Control in Type 2 Diabetes
}

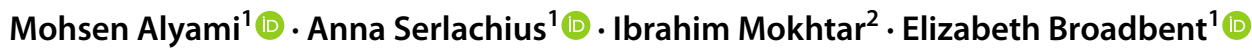

Accepted: 6 September 2021 / Published online: 14 September 2021

(c) International Society of Behavioral Medicine 2021

\begin{abstract}
Background Cross-sectional research demonstrates associations between illness perceptions and glycemic control in people with type 2 diabetes (T2D). Prospective studies are limited and show mixed findings. This study aimed to investigate (1) whether baseline illness perceptions predicted glycemic control (HbA1c levels) at 6-12-month follow-up and (2) possible differences in baseline illness perceptions between individuals who completed at least one HbA1c blood test during the 12-month follow-up and those who did not.

Methods A total of 115 individuals with T2D were recruited from an outpatient clinic. Demographic and clinical information and illness perceptions were assessed at baseline. HbA1c was assessed at baseline and 12 months later from clinical records. Hierarchical multiple linear regression examined associations between baseline illness perceptions and HbA1c levels at 6-12-month follow-up, controlling for age, sex, education, types of diabetes medication, and baseline HbA1c.

Results Univariate analysis showed perceived weight management effectiveness at baseline was associated with lower HbA1c at follow-up ( $r h o=-.25, p=.04, n=67$ ). Adjusted multiple regression showed that HbA1c at baseline was the only significant predictor of HbA1c at $6-12$-month follow-up $(\beta=0.51, p<.001)$. There were no significant differences in baseline illness perceptions between individuals who completed HbA1c blood tests during follow-up $(n=78)$ and those who $\operatorname{did} \operatorname{not}(n=34), p>.05$.

Conclusion Illness perceptions at baseline did not predict longitudinal HbA1c in adjusted analyses, nor completion of HbA1c tests. Results may be due to temporal variability in HbA1c and barriers to accessing blood tests.
\end{abstract}

Keywords Glycemic control $\cdot$ HbA1c $\cdot$ Illness perceptions $\cdot$ Type 2 diabetes

\section{Introduction}

Type 2 diabetes (T2D) accounts for 90 to $95 \%$ of all cases of diabetes. T2D is caused by relative deficiency of insulin secretion by the beta cells and insulin resistance, resulting in hyperglycemia (elevated glucose levels) [1]. Landmark trials in T2D have demonstrated that strict glycemic control can reduce the risk of diabetes-related complications [2]. Glycemic control is assessed using the glycosylated hemoglobin

Elizabeth Broadbent

e.broadbent@auckland.ac.nz

1 Department of Psychological Medicine, Faculty of Medical and Health Sciences, The University of Auckland, Auckland, New Zealand

2 Diabetes and Endocrine Centre, King Khaled Hospital, Najran, Saudi Arabia
A1c test (HbA1c), which reflects the average blood glucose level over approximately 3 months [1].

The frequency of HbA1c testing varies based on illness progression, treatment regimen, and clinician judgement. The American Diabetes Association (ADA) recommends that individuals with diabetes whose HbA1c levels are within the target range perform $\mathrm{HbA} 1 \mathrm{c}$ tests twice a year, whereas those with $\mathrm{HbA1c}$ above the target range or are on intensive treatment regimens should perform $\mathrm{HbAlc}$ test every three months [1]. Guidelines also recommend individuals with diabetes test their blood glucose levels (selfmonitoring of blood glucose, SMBG), especially those with type 1 diabetes (T1D) and insulin-treated T2D. Results from such daily testing can be used to monitor treatment efficacy and inform adjustments to medications, diet, and exercise [1].

A growing body of psychological research based on Leventhal's common sense model of self-regulation (CSM) [3, 
4] has shown that the way people perceive an illness (illness perceptions) play a role in how they cope with their illness [5-8]. These perceptions are grouped into five core domains, namely, illness identity (label and symptoms), timeline (perceived duration), consequences (perceived effects), controllability (whether the illness can be cured or controlled via treatment), and perceived causes. In parallel, individuals also form emotional representations of their illness. The CSM postulates that illness perceptions and emotional representations guide coping processes (e.g., self-care behaviors), which in turn affect health outcomes. In the CSM, people are active problem solvers; they evaluate the effects of their behaviors on their illness and modify their perceptions and coping processes accordingly [3].

Previous reviews have shown that illness perceptions are associated with glycemic control in people with diabetes [9, 10]. Individuals who perceive they can control their diabetes tend to have more optimal glycemic control, whereas individuals who perceive diabetes to have severe consequences, many symptoms, a cyclical timeline, and who are more concerned and emotionally distressed tend to have suboptimal glycemic control [10]. In addition, a recent review concluded that illness perceptions are modifiable through theoretically informed interventions that incorporate behavioral change techniques, with personal and treatment control, coherence, and chronic timeline perceptions being the most likely to change in individuals with T2D [11].

Most of the evidence linking illness perceptions to glycemic control in diabetes is from cross-sectional research. Four longitudinal studies have investigated this relationship and shown mixed results. Reasons for discrepancies in findings may be related to whether the studies adjusted for baseline $\mathrm{HbA1c}$ or not. Analyses adjusted for baseline HbA1c may be more reflective of changes in HbA1c levels over time, whereas analyses not controlling for baseline $\mathrm{HbA} 1 \mathrm{c}$ predict absolute levels and results may be more similar to crosssectional analyses. Other reasons for differences in results between studies may include whether the data has been taken from intervention trials, the type of diabetes patients have, and the timeframe over which the studies were conducted.

In terms of predicting $\mathrm{HbAlc}$ at follow-up, one study with older adults with T2D found that greater treatment effectiveness perceptions and perceiving one's actions in the past had caused their diabetes at baseline predicted more optimal glycemic control levels at 4-month follow-up [12]. However, this study did not control for baseline glycemic control levels [12]. A similar study with older adults with T1D or T2D (who participated in a trial to improve dietary self-management) assessed illness perceptions and $\mathrm{HbA} 1 \mathrm{c}$ at baseline, 3-, 9-, and 12-month follow-up [13]. This study found that greater personal control perceptions at 9 months predicted more optimal glycemic control levels at 12-month follow-up in the adjusted analyses (controlled for baseline glycemic control levels, age, sex, comorbidities, type of diabetes, and allocation group) [13]. These studies highlight the importance of personal and treatment control perceptions in predicting more optimal glycemic control. The importance of control perceptions is consistent with findings from crosssectional studies [10, 14-17].

The two further longitudinal studies found that baseline illness perceptions predicted psychological outcomes but not glycemic control at follow-up. The first study included individuals recently diagnosed with $\mathrm{T} 2 \mathrm{D}$ (who participated in a multicenter cluster trial and assessed at baseline, 4, 8, 12, and 36 months), and found the perceived impact of diabetes at 4 months predicted depression and distress symptoms at 36-month follow-up in the adjusted analyses (controlled for demographic variables and outcomes values at 4 months). In this study, however, illness perceptions at 4 months did not predict glycemic control levels at 36-month follow-up [18]. A further longitudinal study with adults with T1D reported that baseline consequences and personal control perceptions were not related to changes in glycemic control levels over a 5-year period using cross-lagged analysis [19]. Instead, analysis showed that greater personal control perceptions at baseline predicted a relative decrease in self-reported treatmentrelated problems, and greater consequences perceptions at baseline predicted a relative increase in treatmentand food-related, emotional, and social support problems and depressive symptoms 5 years later in the adjusted analyses [19]. Together, these two studies suggest that control and consequences perceptions may predict diabetes-specific distress and depressive symptoms but not $\mathrm{HbAlc}$ or changes in $\mathrm{HbA1c}$ levels.

There is no prospective research exploring whether illness perceptions are related to the frequency of $\mathrm{HbA1c}$ testing (a measure of adherence to $\mathrm{HbA} 1 \mathrm{c}$ testing recommendations). Available evidence from cross-sectional studies shows mixed results regarding the relationship between illness perceptions and SMBG [20]. Perceiving treatment as effective $[21,22]$ and perceiving diabetes as a chronic condition [23] were associated with more frequent SMBG. Other studies, however, did not find these associations [12, 24, 25].

There is a need for further longitudinal studies to examine the relationship between illness perceptions and glycemic control to help to establish the temporality of associations. We conducted a 12-month follow-up study of a sample of individuals with T2D to investigate these associations. In a previous analysis of cross-sectional data from this sample, lower perceptions that insulin therapy could control diabetes and more cyclical timeline perceptions were associated with higher HbA1c levels [26].

This prospective observational study aimed to (1) investigate whether baseline illness perceptions predicted glycemic control in this sample of individuals with T2D at 6-12month follow-up adjusted for baseline levels of $\mathrm{HbA1c}$, and 
(2) investigate possible differences in baseline illness perceptions between individuals who completed at least one HbA1c blood test during the 12-month follow-up and those who did not. Based on previous work [12], it was hypothesized that treatment control perceptions would longitudinally predict glycemic control. No clear hypotheses were developed on how baseline illness perceptions may differ between the two groups given the lack of research exploring this question.

A novel aspect of this study is that it was conducted with Saudi adults with T2D, an underrepresented group in the behavioral medicine literature. It adds to the few longitudinal studies conducted to date in the USA, UK, and Belgium.

\section{Methods}

This study used a prospective observational design. The method of baseline data collection has been reported in full elsewhere [26]. In brief, participants were recruited using convenience sampling from a diabetes outpatient clinic in February and March 2019 in Najran, Saudi Arabia. Participants were included if they were 18 years of age or older, had a formal T2D diagnosis for 1 year or longer, and were prescribed medication to manage their diabetes. Participants were excluded if they were pregnant or too ill on the day of recruitment. Participants did not receive any form of compensation. This study was approved by King Fahad Medical City Institutional Review Board (IRB 20-405E) to extract $\mathrm{HbA1c}$ results from medical charts in June 2020. All participants provided written consent in the original cross-sectional study in 2019 (IRB 18-353E) [26].

The sample size was calculated using $\mathrm{G}^{*}$ Power software [27] based on previous cross-sectional research with South Asian individuals with T2D [28]. The current study was powered to detect a correlation of 0.26 between personal control perceptions and $\mathrm{HbA} 1 \mathrm{c}$ with $80 \%$ power and 0.05 significance level.

\section{Measures}

At baseline, participants provided information regarding age, sex, marital status, educational level, employment status, and monthly income and completed the Brief Illness Perception Questionnaire. Clinical data including body mass index (BMI), duration of diabetes, type and number of diabetes medications, number of comorbid conditions, and diabetesrelated complications were extracted from medical charts at baseline.

\section{IIIness Perceptions}

The Arabic version of the Brief Illness Perception Questionnaire (B-IPQ) was used to measure participants' perceptions of T2D [29]. The questionnaire includes nine items, with the first eight items assessing cognitive and emotional perceptions (personal control, treatment control, coherence, consequences, timeline, illness identity, concern, and emotional response) [14]. These items are scored using a scale from 0 to 10 , with higher scores indicating stronger perceptions. The last item, an open-ended question, requires participants to list the three most likely causes of T2D [14]. Participants' responses were categorized into four groups (psychosocial factors, behavioral factors, hereditary factors, and God's will). The original English version [5, 14] and the Arabic version of the B-IPQ [29] have shown robust psychometric properties.

Six items were added to the B-IPQ based on previous research. One item asked about perceptions of the cyclical nature of T2D ("How much do your diabetes symptoms change from day to day?") and scored using a scale, where $0=$ very stable and $10=$ very changeable. This item was based on the cyclical timeline domain, which is measured in the Revised Illness Perception Questionnaire [30], and previous research has shown to have a negative association with glycemic control [10]. The remaining five items asked about perceptions regarding the effectiveness of self-care behaviors ("How much do you think your [oral medications; insulin injections; healthy diet; weight management; and physical activity] can help your diabetes?') [15, 31]. Format and scoring of these questions were similar to the original B-IPQ items, where $0=$ not at all and $10=$ extremely helpful. Higher scores indicate stronger perceptions.

\section{Glycemic Control (HbA1c Levels)}

Participants' HbA1c levels were extracted from medical charts at baseline and 12-month follow-up. HbA1c levels of $8 \%$ or greater indicated suboptimal glycemic control [1].

\section{Statistical Analysis}

Data were analyzed using IBM SPSS Statistics (version 27). Data were assessed for normality and homoscedasticity using Kolmogorov-Smirnov and Levene's tests. Pearson correlations, $t$-tests, and one-way ANOVAs were conducted for relationships between demographic and clinical variables and $\mathrm{HbA1c}$ at 6-12-month follow-up. Because illness perception item scores were not normally distributed, Spearman correlation analyses were conducted when exploring correlations between baseline illness perceptions and $\mathrm{HbA} 1 \mathrm{c}$ at 6-12-month follow-up. One-way ANOVAs were used to explore the baseline causal beliefs (categorical) and $\mathrm{HbA} 1 \mathrm{c}$ at 6-12-month follow-up. A hierarchical multiple linear regression was conducted to examine whether baseline illness perceptions predicted $\mathrm{HbA} 1 \mathrm{c}$ levels at follow-up, adjusting for age, sex, education, type of diabetes medication, and 
baseline HbA1c. For the above analyses, only participants with $\mathrm{HbA} 1 \mathrm{c}$ data between 6 and 12 months after baseline were included. A series of Mann-Whitney $U$ and chi-square tests were used to compare baseline illness perceptions and causal beliefs between participants who completed at least one $\mathrm{HbA} 1 \mathrm{c}$ blood test during the 12-month follow-up period and those who did not. Participants with missing data were excluded from the analysis, hence sample sizes varied across analyses as reported in the results. Statistical significance was set at $p<0.05$ (two-tailed).

\section{Results}

Figure 1 shows participants' enrolment from recruitment to follow-up. Of the 173 individuals approached in the initial cross-sectional study, 46 individuals did not meet the inclusion criteria. Of the 127 eligible participants, 115 participants provided informed consent to participate [26]. Three participants died during the 12-month follow-up and hence were excluded from the analysis. The final analytical sample at followup consisted of 112 participants, of whom 67 participants had HbA1c blood test results within 6-12 months after baseline, whereas 11 participants had HbA1c blood test results available in less than 6 months after baseline. Thirty-four participants did not have any $\mathrm{HbAlc}$ blood test results available in their medical charts during the 12-month follow-up.

The mean age was 56 years (SD 12.38), with $58 \%$ male participants. The mean BMI was 30.89 (SD 5.11), and the mean time since diagnosis was 10.16 years (SD 7.12). More than half of participants $(56 \%)$ were prescribed a combination of oral medications and insulin therapy, with $43 \%$ of participants taking three or more diabetes medications. Significant comorbidities, including hypertension and dyslipidemia, were common, and $20 \%$ of participants had two or more comorbid conditions. Complications were
Fig. 1 Flow chart of participants throughout the study. The analytical sample included participants with $\mathrm{HbA1c}$ data $<6$ months after baseline $(n=11)$, participants with HbA1c within 6-12 months after baseline $(n=67)$, and participants without $\mathrm{HbA} 1 \mathrm{c}$ data after baseline $(n=34)$
Assessed for eligibility $(\mathrm{n}=173)$

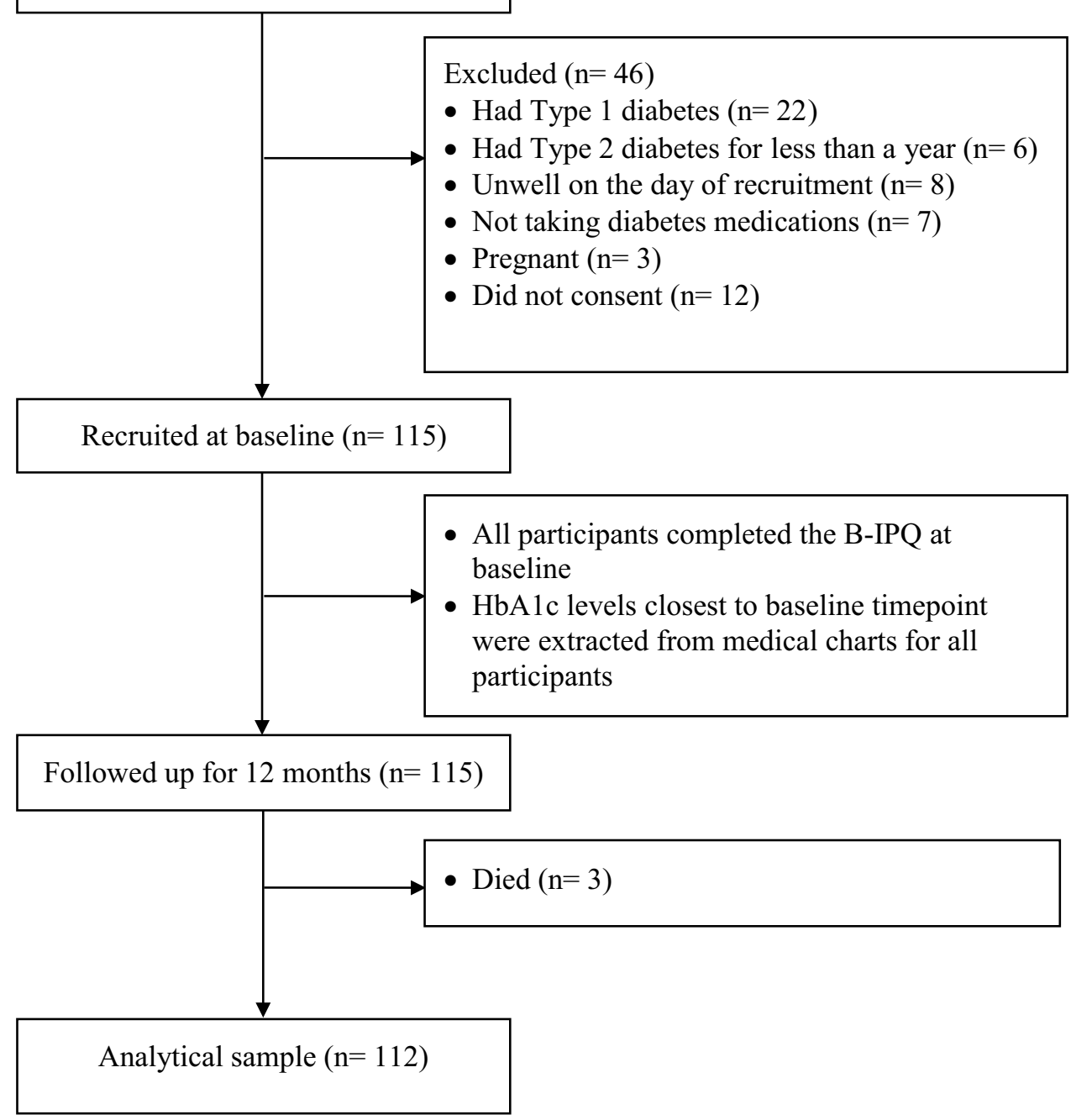


also common among participants, including coronary heart disease, retinopathy, and nephropathy, with 39\% of participants having two or more complications. Sample characteristics at baseline are shown in Table 1.
The mean HbA1c level at baseline for the whole sample was $8.72 \%$ (SD 1.38), with $67 \%$ of participants exhibiting suboptimal glycemic control (HbA1c $>8 \%)$. Over the 12-month follow-up, mean HbA1c slightly decreased for

Table 1 Demographic and clinical characteristics of the sample, stratified by whether or not participants had HbA1c data at follow up

\begin{tabular}{|c|c|c|c|c|}
\hline \multirow[t]{2}{*}{ Percentage $(n)$ unless stated otherwise } & \multirow{2}{*}{$\begin{array}{l}\text { Total sample at follow-up } \\
n=112\end{array}$} & \multicolumn{2}{|c|}{ Follow-up HbA1c data available } & \multirow{2}{*}{$\begin{array}{l}\text { Difference } \\
\left(t \text {-test or } \chi^{2}\right)\end{array}$} \\
\hline & & Yes $(n=78)$ & No $(n=34)$ & \\
\hline Age (mean/SD) & $56.05(12.38)$ & $57.13(12.42)$ & $53.59(12.11)$ & $-1.40^{\mathrm{NS}}$ \\
\hline Sex & & & & $0.28^{\mathrm{NS}}$ \\
\hline Male & $58 \%(65)$ & $56 \%(44)$ & $62 \%(21)$ & \\
\hline Female & $42 \%(47)$ & $44 \%(34)$ & $38 \%(13)$ & \\
\hline Marital status & & & & $0.02^{\mathrm{NS}}$ \\
\hline Married & $71 \%(80)$ & $72 \%(56)$ & $71 \%(24)$ & \\
\hline Not married & $29 \%(32)$ & $28 \%(22)$ & $29 \%(10)$ & \\
\hline Education level & & & & $7.16^{\mathrm{NS}}$ \\
\hline Illiterate & $24 \%(27)$ & $27 \%(21)$ & $18 \%(6)$ & \\
\hline Read and write only & $31 \%(34)$ & $34 \%(27)$ & $21 \%(7)$ & \\
\hline High school & $22 \%(25)$ & $22 \%(17)$ & $23 \%(8)$ & \\
\hline Tertiary education & $23 \%(26)$ & $17 \%(13)$ & $38 \%(13)$ & \\
\hline Employment & & & & $0.54^{\mathrm{NS}}$ \\
\hline Employed & $29 \%(32)$ & $27 \%(21)$ & $33 \%(11)$ & \\
\hline Unemployed & $37 \%(42)$ & $37 \%(29)$ & $38 \%(13)$ & \\
\hline Retired & $34 \%(38)$ & $36 \%(28)$ & $29 \%(10)$ & \\
\hline Income $(\mathrm{SAR})^{\mathrm{a}}$ & & & & $2.35^{\mathrm{NS}}$ \\
\hline 10,000 or less & $61 \%(68)$ & $65 \%(51)$ & $50 \%(17)$ & \\
\hline$>10,000$ & $39 \%(44)$ & $35 \%(27)$ & $50 \%(17)$ & \\
\hline BMI (mean/SD) & $30.89(5.11)$ & $30.51(5.07)$ & $31.78(5.16)$ & $2.14^{\mathrm{NS}}$ \\
\hline Normal weight & $13 \%(14)$ & $14 \%(11)$ & $9 \%(3)$ & \\
\hline Overweight & $33 \%(37)$ & $36 \%(28)$ & $26 \%(9)$ & \\
\hline Obese & $54 \%(61)$ & $50 \%(39)$ & $65 \%(22)$ & \\
\hline Baseline $\mathrm{HbA1c}($ mean/SD) & $8.72(1.38)$ & $8.68(1.33)$ & $8.81(1.49)$ & $0.44^{\mathrm{NS}}$ \\
\hline Time since diagnosis (years) (mean/SD) & $10.16(7.12)$ & $10.00(6.85)$ & $10.53(7.81)$ & $0.36^{\mathrm{NS}}$ \\
\hline Type of medication & & & & $0.04^{\mathrm{NS}}$ \\
\hline Oral medication only & $45 \%(51)$ & $46 \%(36)$ & $44 \%(15)$ & \\
\hline Oral medication and insulin therapy & $55 \%(61)$ & $54 \%(42)$ & $56 \%(19)$ & \\
\hline Number of medications & & & & $4.25^{\mathrm{NS}}$ \\
\hline One & $4 \%(5)$ & $6 \%(5)$ & $0 \%$ & \\
\hline Two & $53 \%(59)$ & $54 \%(42)$ & $50 \%(17)$ & \\
\hline Three or more & $43 \%(48)$ & $40 \%(31)$ & $50 \%(17)$ & \\
\hline Number of complications & & & & $1.70^{\mathrm{NS}}$ \\
\hline None & $29 \%(32)$ & $32 \%(25)$ & $21 \%(7)$ & \\
\hline One & $32 \%(36)$ & $29 \%(23)$ & $38 \%(13)$ & \\
\hline Two or more & $39 \%(44)$ & $39 \%(30)$ & $41 \%(14)$ & \\
\hline Number of comorbidities & & & & $1.82^{\mathrm{NS}}$ \\
\hline None & $36 \%(40)$ & $40 \%(31)$ & $26 \%(9)$ & \\
\hline One & $44 \%(49)$ & $36 \%(28)$ & $62 \%(21)$ & \\
\hline Two or more & $20 \%(23)$ & $24 \%(19)$ & $12 \%(4)$ & \\
\hline
\end{tabular}

$N S$ not significant

${ }^{\text {a }}$ Saudi Riyal 
Table 2 Glycemic control as measured by glycosylated hemoglobin (HbA1c) levels at baseline and follow up

\begin{tabular}{|c|c|c|c|c|}
\hline & Mean (SD) at baseline & Mean (SD) at follow up & $\begin{array}{l}\text { Mean change in } \\
\text { glycemic control }\end{array}$ & $\begin{array}{l}\text { Suboptimal } \\
\text { glycemic control } \\
(\geq 8 \%)\end{array}$ \\
\hline & & & & $\%, n$ \\
\hline Whole sample $(n=112)$ & $8.72 \%(1.38)$ & & & $67.86 \%(76)$ \\
\hline Participants with HbA1c data after baseline $(n=78)^{\mathrm{a}}$ & $8.68 \%(1.33)$ & $8.48 \%(1.63)$ & $0.20 \%(1.36)$ & $57.69 \%(45)$ \\
\hline $\begin{array}{l}\text { Participants with } \mathrm{HbA1c} \text { data within } 6-12 \text { months } \\
\text { after baseline }(n=67)\end{array}$ & $8.63 \%(1.36)$ & $8.52 \%(1.70)$ & $0.11 \%(1.45)$ & $58.21 \%(39)$ \\
\hline
\end{tabular}

${ }^{\mathrm{a}}$ This sample included participants with HbA1c data $<6$ months after baseline $(n=11)$ and those with HbA1c data within $6-12$ months after baseline $(n=67)$

participants who had $\mathrm{HbA} 1 \mathrm{c}$ blood tests during the 6-12month follow-up; $t(66)=-0.64, p=0.53$ ), equating to a mean decrease of $0.11 \%$ as shown in Table 2.

\section{Predicting $\mathrm{HbA} 1 \mathrm{c}$ at Follow-Up from Baseline Illness Perceptions}

None of the demographic variables was associated with HbA1c levels at follow-up $(p>0.05)$. Of the clinical variables, type of diabetes medication was associated with HbA1c levels $(r=0.28, p=0.02)$. Participants who were prescribed a combination of oral and insulin therapy (mean HbA1c 8.93, SD 1.58) compared with those who were only prescribed oral medications (mean HbA1c 7.98, SD 1.73) had significantly higher HbA1c at follow-up $t(65)=-2.33$, $p=0.02$. As expected, $\mathrm{HbA} 1 \mathrm{c}$ at baseline was associated with $\mathrm{HbA} 1 \mathrm{c}$ at follow-up $(r=0.57, p<0.001)$.

More cyclical timeline perceptions (Spearman's $r h o=0.20, p=0.03)$ and lower perceptions that insulin therapy could control T2D $(r=-0.26, p=0.04)$ at baseline were associated with higher $\mathrm{HbA} 1 \mathrm{c}$ levels at baseline (as reported earlier [26]). However, illness perceptions were not associated with $\mathrm{HbA1c}$ at 6-12-month follow-up, except for perception of weight management effectiveness (Table 3 ). Perceiving weight management as an effective approach to controlling T2D was associated with lower HbA1c at followup $(r=-0.25, p=0.04)$.

A series of one-way ANOVAs were conducted to examine the relationships between reported causal beliefs at baseline and $\mathrm{HbA} 1 \mathrm{c}$ levels at follow-up. Of the 67 participants with $\mathrm{HbA1c}$ data at 6-12-month follow-up, 46 participants answered the causal B-IPQ item at baseline. There were no significant differences in HbAlc levels at 6-12-month follow-up between participants who cited psychosocial factors, behavioral factors, hereditary factors, or God's will as causing their diabetes and those who did not (Table 4).

In the hierarchical multiple linear regression with $\mathrm{HbAlc}$ at follow-up as the dependent variable, covariates including age, sex, education, type of diabetes medication, and baseline HbA1c were entered in Model 1 and perceptions of weight management effectiveness were added in Model 2 (Table 5). Model 1 was significant $(p<0.001)$ and accounted for $36.6 \%$ of the total variance in HbA1c levels at follow-up. In terms of individual predictors, only baseline $\mathrm{HbA} 1 \mathrm{c}$ was associated with higher HbA1c levels at follow-up $(\beta=0.51$, $p<0.001)$. Model 2 was also significant $(p<0.001)$, explaining $36.8 \%$ of the total variance in HbA1c levels at followup. Perceptions of weight management effectiveness did not improve the prediction model, $R^{2} \Delta=0.001, p=0.74$. Baseline $\mathrm{HbA} 1 \mathrm{c}$ remained the only significant predictor of higher HbA1c at follow-up $(\beta=0.51, p<0.001)$.

A series of Mann-Whitney $U$ and chi-square tests were conducted to examine whether participants who completed at least one HbA1c blood test during the 12-month followup $(n=78)$ and those who did not $(n=34)$ differed in terms of baseline illness perceptions and causal beliefs. The two

Table 3 Spearman correlations between baseline illness perceptions and HbA1c at baseline $(n=115)$ and 6-12 months follow up $(n=67)$

\begin{tabular}{|c|c|c|c|c|}
\hline \multirow[t]{2}{*}{ Baseline perceptions } & \multicolumn{2}{|c|}{$\begin{array}{l}\mathrm{HbA1c} \text { at } \\
\text { baseline }\end{array}$} & \multicolumn{2}{|c|}{$\begin{array}{l}\text { HbA1c at fol- } \\
\text { low up }\end{array}$} \\
\hline & $r$ & $p$ value & $r$ & $p$ value \\
\hline Consequences & .11 & .26 & -.06 & .62 \\
\hline Timeline (acute/chronic) & .09 & .36 & .08 & .53 \\
\hline Personal control & -.07 & .47 & .05 & .70 \\
\hline Treatment control & -.10 & .27 & .06 & .65 \\
\hline Illness identity & .10 & .28 & -.05 & .67 \\
\hline Concerns & .12 & .21 & -.06 & .64 \\
\hline Coherence & .08 & .42 & -.06 & .61 \\
\hline Emotional response & .03 & .73 & -.14 & .26 \\
\hline Cyclical timeline & .20 & .03 & -.01 & .93 \\
\hline Oral medication effectiveness & .002 & .98 & -.06 & .66 \\
\hline Insulin effectiveness ${ }^{\mathrm{a}}$ & -.26 & .04 & -.15 & .36 \\
\hline Diet effectiveness & -.01 & .93 & -.12 & .33 \\
\hline Weight management effectiveness & -.14 & .13 & -.25 & .04 \\
\hline Physical activity effectiveness & -.09 & .35 & -.19 & .12 \\
\hline
\end{tabular}

${ }^{\mathrm{a}} 62$ and 38 total valid responses at baseline and follow-up, respectively, because only participants prescribed insulin completed this item 
Table 4 One-way ANOVA analyses between causal beliefs at baseline and $\mathrm{HbA} 1 \mathrm{c}$ levels at 6-12 month follow-up $\left(n=46^{\mathrm{a}}\right)$

\begin{tabular}{lllllllll}
\hline Perceived causes & \multicolumn{2}{l}{ Belief endorsed } & & \multicolumn{3}{l}{ Belief not endorsed } & & \\
\cline { 2 - 3 } & $n$ & HbA1c Mean (SD) & & & HbA1c Mean $(\mathrm{SD})$ & $F$ statistic, significance & $\eta^{2}$ \\
\hline Psychosocial factors & 2 & $7.60(0.14)$ & 44 & $8.49(1.73)$ & & $F(1,44)=0.52, p=.48$ & .012 \\
Behavioral factors & 24 & $8.57(1.70)$ & 22 & $8.31(1.73)$ & & $F(1,44)=0.26, p=.61$ & .006 \\
Hereditary factors & 32 & $8.48(1.68)$ & 14 & $8.36(1.80)$ & & $F(1,44)=0.05, p=.83$ & .001 \\
God's will & 13 & $8.89(1.58)$ & 33 & $8.27(1.73)$ & & $F(1,44)=1.25, p=.27$ & .028 \\
\hline
\end{tabular}

$\eta^{2}$ eta squared

${ }^{a}$ Only 46 of the 67 participants with $\mathrm{HbA1c}$ data at 6-12 months follow-up answered the causal B-IPQ item at baseline groups did not differ in any of the demographic and clinical variables (Table 1). There were no significant differences in any of the illness perceptions or causal beliefs between the two groups $(p>0.05)$ (Tables S1 \& S2 in Electronic Supplementary Material).

\section{Discussion}

This study examined (1) whether baseline illness perceptions predicted glycemic control at 12-month follow-up among individuals with T2D and (2) examined differences in baseline illness perceptions between individuals who had an $\mathrm{HbA} 1 \mathrm{c}$ test done during the 12-month follow-up and those who did not. The findings did not support the hypothesis that baseline treatment control perceptions would predict glycemic control at follow-up. Of all the treatment perceptions (oral medication, insulin therapy, physical exercise, healthy eating, and weight management), only perceiving weight management as an effective approach to controlling T2D at baseline was associated with lower HbA1c at 6-12-month follow-up. This relationship, however, was not significant when controlling for age, sex, education, type of diabetes medication, and baseline HbA1c in the regression analysis. Baseline illness perceptions and causal beliefs did not significantly differ between individuals who had an HbA1c blood test done during the 12-month follow-up and those who did not.

One reason for the non-existent longitudinal associations between illness perceptions and glycemic control may be due to reduced power caused by attrition and a high number of participants without HbA1c blood tests during the

Table 5 Hierarchical multiple linear regression with $\mathrm{HbA1c}$ at follow-up as the dependent variable $(n=67)$

\begin{tabular}{|c|c|c|c|c|c|c|}
\hline \multirow[t]{2}{*}{ Predictor } & \multicolumn{3}{|l|}{ Model 1} & \multicolumn{3}{|l|}{ Model 2} \\
\hline & $B(\mathrm{SE})$ & $\beta$ & $95 \% \mathrm{CI}$ & $B(\mathrm{SE})$ & $\beta$ & $95 \% \mathrm{CI}$ \\
\hline Age & $-0.01(0.02)$ & -0.09 & $-0.05,0.03$ & $-0.01(0.02)$ & -0.11 & $-.06,0.03$ \\
\hline Sex (Male) & $-0.8(0.39)$ & -0.02 & $-0.85,0.69$ & $-0.07(0.39)$ & -0.02 & $-0.85,0.72$ \\
\hline \multicolumn{7}{|l|}{ Education } \\
\hline Illiterate & $-0.23(0.77)$ & -0.06 & $-1.78,1.32$ & $-0.19(0.79)$ & -0.05 & $-1.77,1.38$ \\
\hline Read and write only & $-0.26(0.62)$ & -0.07 & $-1.50,0.97$ & $-0.26(0.62)$ & -0.07 & $-1.51,0.99$ \\
\hline High school & $0.32(0.61)$ & 0.08 & $-0.92,1.54$ & $0.31(0.62)$ & 0.08 & $-0.94,1.55$ \\
\hline Tertiary education & Reference group & & & Reference group & & \\
\hline Type of medications & $0.29(0.40)$ & 0.09 & $-0.52,1.10$ & $0.28(0.431$ & 0.08 & $-0.54,1.09$ \\
\hline Baseline HbA1c & $0.68^{*}(0.15)$ & 0.51 & $0.35,0.93$ & $0.63^{*}(0.15)$ & 0.51 & $0.34,0.93$ \\
\hline \multicolumn{7}{|l|}{ B-IPQ } \\
\hline $\begin{array}{l}\text { Weight management } \\
\text { effectiveness }\end{array}$ & & & & $-0.02(0.07)$ & -0.04 & $-0.17,0.12$ \\
\hline$R^{2}$ & 0.366 & & & & & 0.368 \\
\hline$F$ statistic & $F(7,59)=4.87, p<.001$ & & & $F(8,58)=4.21, p<.001$ & & \\
\hline$R^{2} \Delta$ & & & & & & 0.001 \\
\hline$F \Delta$ & & & & $F \Delta(1,58)=0.11, p=.74$ & & \\
\hline
\end{tabular}

$B$-IPQ Brief Illness Perception Questionnaire, $B$ unstandardized coefficient, $S E$ standard error, $\beta$ standardized beta coefficients, $C I$ confidence interval, $R^{2} R$ square; $R^{2} \Delta R$ square change, $F \Delta F$ change

${ }^{*} p<.001$ 
follow-up. To detect a small-medium size effect $(r=0.26)$ with $80 \%$ power and 0.05 alpha in a multiple linear regression at follow-up (with 12 predictors), our sample of 112 participants would have been optimal. However, about $41 \%$ of our sample was lost to follow-up (either died, had HbA1c data in less than 6 months after baseline, or did not have $\mathrm{HbA1c}$ data after baseline at all). Another possible reason is that $\mathrm{HbA} 1 \mathrm{c}$ is variable over time that perceptions are dependent on current $\mathrm{HbA} 1 \mathrm{c}$ levels. It could be that current symptoms, HbA1c levels, and complications are driving perceptions rather than the other way around. It is important to note that in the CSM, people are active problem solvers and the results of their actions feedback to inform perceptions and coping processes.

Previous literature on illness perceptions has highlighted the importance of differentiating between "hard" outcomes (e.g., HbA1c) and "softer" outcomes (e.g., quality of life) [32]. This came as a result of findings of a meta-analytical review that found illness perceptions were often strongly associated with psychological outcomes such as psychological distress and wellbeing but unrelated or weakly related to $\mathrm{HbA1c}$ [9].

There is only limited longitudinal research, based on the CSM, investigating the relationships between illness perceptions and subsequent glycemic control in diabetes. Our findings are consistent with previous studies that did not find associations between baseline illness perceptions and subsequent glycemic control in individuals with T2D at 3 years [18] or changes in glycemic control at 5 year follow-up in the adjusted analyses [19]. However, our findings contrast with other research that reported higher treatment effectiveness [12] and control perceptions [13] predicted lower subsequent $\mathrm{HbA} 1 \mathrm{c}$ levels in older individuals with diabetes after three or four months respectively. This suggests longitudinal associations may weaken with time.

It has been suggested that beliefs about the effectiveness of self-care behaviors (e.g., exercise and weight management) could be more important than perceptions about the illness itself as they have been shown to explain more variance above and beyond that explained by illness perceptions in a 1-year prospective study [31]. In this study, individuals who believed that physical activity, eating smaller quantities of food, and avoiding fatty foods were important in controlling diabetes reported greater subsequent exercise levels. This study, however, did not investigate the longitudinal associations between illness perceptions, beliefs about selfcare behaviors, and glycemic control [31].

Furthermore, causal beliefs as assessed by the B-IPQ were not associated with $\mathrm{HbA} 1 \mathrm{c}$ at follow-up in the present study, similar to results from the cross-sectional analysis [26]. A review previously noted that illness perception research in diabetes and other conditions has often omitted the B-IPQ item on causal beliefs [5]. Previous cross-sectional research has shown that causal beliefs are related to adherence to self-care behaviors $[25,33]$. In one prospective study, believing one's actions in the past had caused their diabetes (personal responsibility for causing diabetes) was associated with lower HbA1c four months later in older adults, more in females than males; however, this study did not control for baseline HbA1c [12].

Several explanations could be highlighted to explain the differences in the longitudinal results from the present study and the Hampson et al. studies. First, although Hampson et al. [12] included individuals with T2D, the sample was older, predominantly female, with the majority having at least high school level education, lower baseline $\mathrm{HbAlc}$, and shorter duration of diabetes in contrast to the current sample. The Hampson et al. [12] also did not collect HbA1c at baseline and therefore did not control for it in the analyses. Second, in their other study, Hampson et al. [13] included older individuals with T1D and T2D with lower mean HbA1c at baseline (mean HbA1c 7.92\%) compared to our sample (mean HbA1c 8.72\%). Recent research has also shown that illness perceptions may differ between the two types of diabetes, with worse and more threatening perceptions of diabetes in individuals with T2D than individuals with T1D [23]. Third, the Hampson et al. studies [12, 13] investigated the relationship between illness perceptions and glycemic control in a short term (over 3 and 4 months), whereas participants in the current study were followed for 12 months. Finally, differences might also be explained by the fact that the Hampson et al. studies $[12,13]$ used the Personal Models of Diabetes Interview (PMDI) [22], whereas we used the B-IPQ [14] to assess illness perceptions. The B-IPQ is a theoretically informed measure that has evolved over time to incorporate new evidence regarding the importance of other perception domains. The B-IPQ is widely used in diabetes research and has demonstrated good psychometric properties yet may have reduced variance [5].

Illness perceptions and causal beliefs did not significantly differ between individuals who had at least one $\mathrm{HbA} 1 \mathrm{c}$ blood test done during the 12-month follow-up and those who did not. This finding is consistent with earlier research [34] but contrary to other research that showed illness perceptions were associated with clinic non-attendance. For example, in T1D, shorter timeline, lower personal and treatment control, and more severe consequence perceptions were associated with clinic non-attendance [35]. Another study in T1D and T2D found that worse emotional response was associated with an increased risk of clinic non-attendance [36].

It is concerning that about a third of participants in the current study did not have any HbA1c blood tests recorded in their medical charts during the 12-month follow-up. It is recommended that individuals with diabetes perform at least two HbA1c blood tests annually [1]. The lack of test results may indicate inadequate adherence to $\mathrm{HbA} 1 \mathrm{c}$ testing 
recommendations and/or clinic non-attendance. Other reasons for lack of test results may include treating clinicians forgetting to give blood test referrals, patient forgetfulness, lack of transportation, or busy work schedules. It is also worth noting that $\mathrm{HbA} 1 \mathrm{c}$ follow-up data were collected during the COVID-19 pandemic, where the first confirmed case in Saudi Arabia was reported on March 2, and by June 28, 2020 , there were 182,493 confirmed cases and 1551 deaths [37]. Therefore, it is possible that COVID-19 restrictions may have prevented some individuals from attending their diabetes clinic appointments in this study.

Clinic non-attendance is a common problem in diabetes $[38,39]$. It is associated with inadequate adherence to medication and self-monitoring of blood glucose and suboptimal glycemic control, which all contribute to life-threatening diabetes-related complications [40]. Systematic reviews have identified several factors associated with clinic nonattendance, including patient factors (e.g., socioeconomic status, knowledge, health literacy, and health beliefs, and perceptions), disease-related factors (e.g., treatment regimen, comorbidities, and $\mathrm{HbA} 1 \mathrm{c}$ level), and healthcare provider factors (e.g., poor communication, long interval to appointment, and healthcare provider's attitudes and behaviors) [38, 41-43]. Healthcare providers and systems should utilize a range of interventions that are known to reduce clinic nonattendance both at the patient level (e.g., SMS and telephone reminders) and the healthcare system level (e.g., clinician continuity, efficient register, and recall system and online clinics) [38, 41, 44, 45].

There are strengths and limitations to the current study. Strengths of the study lie in its longitudinal design and the use of validated measures to assess illness perceptions and glycemic control. Second, each B-IPQ item was scored and analyzed separately as recommended by the scale's authors, rather than calculating a total score whereby information is lost about the perceptions most strongly linked to outcomes [5]. We also included and analyzed responses on the causal item of the B-IPQ, which research on illness perceptions in diabetes has often omitted. Finally, this study was conducted in Saudi Arabia, where T2D is a major issue and research on illness perceptions is relatively new.

A limitation is that although the initial cross-sectional study was adequately powered, due to attrition and the number of individuals without HbA1c blood test results at follow-up, the sample size was reduced significantly. Furthermore, illness perceptions were assessed only at baseline, and hence, we are unable to comment on how illness perceptions changed over time and whether these changes were related to $\mathrm{HbA} 1 \mathrm{c}$ at follow-up. Larger prospective studies are warranted to boost statistical power and extend these findings. Future research should assess whether changes in illness perceptions over time are related to changes in glycemic control over multiple time points in a more representative sample of adults with T2D. This may help to assess the temporality of associations between illness perceptions and HbAlc.

Supplementary Information The online version contains supplementary material available at https://doi.org/10.1007/s12529-021-10024-y.

Acknowledgements We would like to acknowledge and thank King Fahad Medical City and the Diabetes and Endocrine Centre at King Khaled Hospital for their cooperation and support. We would also like to thank our participants for taking part in this study.

Funding The first author (M.A.) is receiving financial support from the Saudi Ministry of Higher Education (King Salman Scholarship Program) and the University of Auckland Student Funds.

\section{Declarations}

Disclaimer Funding agencies were not involved in the design of the study, the collection, analysis and interpretation of data, writing the report, or the decision to submit the report for publication.

Ethical Approval All procedures performed in studies involving human participants were in accordance with the ethical standards of the institutional and/or national research committee and with the 1994 Helsinki declaration and its later amendments or comparable ethical standards.

Informed consent Informed consent was obtained from all individual participants included in the study.

Conflict of interest The authors declare no competing interests.

\section{References}

1. American Diabetes Association. Standards of medical care in diabetes-2020. Diabetes Care. 2020;43(Supplement 1):S1-212.

2. UK Prospective Diabetes Study Group. Intensive blood-glucose control with sulphonylureas or insulin compared with conventional treatment and risk of complications in patients with type 2 diabetes (UKPDS 33). The Lancet. 1998;352(9131):837-53.

3. Leventhal H, Brissette I, Leventhal EA. The common-sense model of self-regulation of health and illness. In: Cameron LD, Leventhal $\mathrm{H}$, editors. The self-regulation of health and illness behaviour. London: Routledge; 2003. p. 42-65.

4. Diefenbach MA, Leventhal H. The common-sense model of illness representation: Theoretical and practical considerations. $\mathbf{J}$ Soc Distress Homeless. 1996;5(1):11-38.

5. Broadbent E, Wilkes C, Koschwanez H, Weinman J, Norton S, Petrie KJ. A systematic review and meta-analysis of the Brief Illness Perception Questionnaire. Psychol Health. 2015;30(11):1361-85.

6. Broadbent E, Schoones JW, Tiemensma J, Kaptein AA. A systematic review of patients' drawing of illness: Implications for research using the common sense model. Health Psychol Rev. 2019;13(4):406-26.

7. Dempster M, Howell D, McCorry NK. Illness perceptions and coping in physical health conditions: A meta-analysis. J Psychosom Res. 2015;79(6):506-13.

8. Hagger MS, Koch S, Chatzisarantis NLD, Orbell S. The common sense model of self-regulation: meta-analysis and test of a process model. Psychol Bull. 2017;143(11):1117-54. 
9. Hagger MS, Orbell S. A meta-analytic review of the commonsense model of illness representations. Psychol Health. 2003;18(2):141-84.

10. Mc Sharry J, Moss-Morris R, Kendrick T. Illness perceptions and glycaemic control in diabetes: A systematic review with meta-analysis. Diabet Med. 2011;28(11):1300-10.

11. Alyami M, Serlachius A, O'Donovan C, Werf B van der, Broadbent E. A systematic review of illness perception interventions in type 2 diabetes: Effects on glycaemic control and illness perceptions. Diabet Med. 2021;38(3):e14495.

12. Hampson SE, Glasgow RE, Foster LS. Personal models of diabetes among older adults: relationship to self-management and other variables. Diabetes Educ. 1995;21(4):300-7.

13. Hampson SE, Glasgow RE, Strycker LA. Beliefs versus feelings: a comparison of personal models and depression for predicting multiple outcomes in diabetes. Br J Health Psychol. 2000;5(1):27-40.

14. Broadbent E, Petrie KJ, Main J, Weinman J. The Brief Illness Perception Questionnaire. J Psychosom Res. 2006;60(6):631-7.

15. Broadbent E, Donkin L, Stroh JC. Illness and treatment perceptions are associated with adherence to medications, diet, and exercise in diabetic patients. Diabetes Care. 2011;34(2):338-40.

16. Martinez K, Lockhart S, Davies M, Lindsay JR, Dempster M. Diabetes distress, illness perceptions and glycaemic control in adults with type 2 diabetes. Psychol Health Med. 2018;23(2):171-7.

17. Voigt A, Madrid E, Pacheco-Huergo V, Rastello A, Castro D, NavarroBrito I, et al. Association of glycaemia with perceived threat of illness in patients with type 2 diabetes. Prim Care Diabetes. 2015;9(6):426-31.

18. Skinner TC, Khunti K, Carey ME, Dallosso H, Heller S, Davies MJ. Stability and predictive utility, over 3 years, of the illness beliefs of individuals recently diagnosed with type 2 diabetes mellitus. Diabet Med. 2014;31(10):1260-3.

19. Rassart J, Luyckx K, Berg CA, Bijttebier P, Moons P, Weets I. Psychosocial functioning and glycemic control in emerging adults with Type 1 diabetes: A 5-year follow-up study. Health Psychol. 2015;34(11):1058-65.

20. Harvey JN, Lawson VL. The importance of health belief models in determining self-care behaviour in diabetes. Diabet Med. 2009;26(1):5-13.

21. Glasgow RE, Hampson SE, Strycker LA, Ruggiero L. Personalmodel beliefs and social-environmental barriers related to diabetes self-management. Diabetes Care. 1997;20(4):556-61.

22. Hampson SE, Glasgow RE, Toobert DJ. Personal models of diabetes and their relations to self-care activities. Health Psychol. 1990;9(5):632-46.

23. Abubakari A-R, Cousins R, Thomas C, Sharma D, Naderali EK. Sociodemographic and clinical predictors of self-management among people with poorly controlled type 1 and type 2 diabetes: the role of illness perceptions and self-efficacy. J Diabetes Res. 2016;2016:6708164.

24. Abubakari A-R, Jones MC, Lauder W, Kirk A, Anderson J, Devendra D. Associations between knowledge, illness perceptions, self-management and metabolic control of type 2 diabetes among African and Europeanorigin patients. J Nurs Healthc Chronic Illn. 2011;3(3):245-56.

25. Alyami M, Serlachius A, Mokhtar I, Broadbent E. The association of illness perceptions and God locus of health control with self-care behaviours in patients with type 2 diabetes in Saudi Arabia. Health Psychol Behav Med. 2020;8(1):329-48.

26. Alyami M, Serlachius A, Mokhtar I, Broadbent E. Illness perceptions, HbA1c, and adherence in type 2 diabetes in Saudi Arabia. Patient Prefer Adher. 2019;13:1839-50.

27. Faul F, Erdfelder E, Lang A-G, Buchner A. G*Power 3: A flexible statistical power analysis program for the social, behavioral, and biomedical sciences. Behav Res Methods. 2007;39(2):175-91.

28. Bean D, Cundy T, Petrie KJ. Ethnic differences in illness perceptions, self-efficacy and diabetes self-care. Psychol Health. 2007;22(7):787-811.
29. Saarti S, Jabbour H, El Osta N, Hajj A, Khabbaz LR. Cross-cultural adaptation and psychometric properties of an Arabic language version of the Brief Illness Perception Questionnaire in Lebanon. Libyan J Med. 2016;11(10):3402.

30. Moss-Morris R, Weinman J, Petrie KJ, Horne R, Cameron LD, Buick D. The Revised Illness Perception Questionnaire (IPQ-R). Psychol Health. 2002;17(1):1-16.

31. French DP, Wade AN, Farmer AJ. Predicting self-care behaviours of patients with type 2 diabetes: the importance of beliefs about behaviour, not just beliefs about illness. J Psychosom Res. 2013;74(4):327-33.

32. Benyamini $\mathrm{Y}$, Leventhal $\mathrm{H}$, et al. Beliefs and perceptions of health and illness. In: Llewellyn C, Ayers S, McManus C, Newman S, Petrie KJ, Revenson TA, et al., editors. Cambridge handbook of psychology, health and medicine. 3rd ed. Cambridge: Cambridge University Press; 2019. p. 106-9.

33. van Puffelen AL, Heijmans MJWM, Rijken M, Rutten GEHM, Nijpels G, Schellevis FG. Illness perceptions and self-care behaviours in the first years of living with type 2 diabetes; does the presence of complications matter? Psychol Health. 2015;30(11):1274-87.

34. Thongsai S. Do illness perceptions predict the attendance rate at diabetic outpatient clinic? Glob J Health Sci. 2015;7(2):254-63.

35. Lawson VL, Bundy C, Lyne PA, Harvey JN. Using the IPQ and PMDI to predict regular diabetes care-seeking among patients with type 1 diabetes. Br J Health Psychol. 2004;9(2):241-52.

36. Eades C, Alexander H. A mixed-methods exploration of nonattendance at diabetes appointments using peer researchers. Health Expect. 2019;22(6):1260-71.

37. Saudi Center for Disease Prevention and Control. Saudi Center for Disease Prevention and Control. 2020. https://covid19.cdc.gov.sa/. Cited 30 Jun 2020.

38. Brewster S, Bartholomew J, Holt RIG, Price H. Non-attendance at diabetes outpatient appointments: a systematic review. Diabet Med. 2020;37(9):1427-42.

39. McComb S, Tian Z, Sands L, Turkcan A, Zhang L, Frazier S, et al. Cancelled primary care appointments: a prospective cohort study of diabetic patients. J Med Syst. 2017;41(4):53.

40. Karter AJ, Parker MM, Moffet HH, Ahmed AT, Ferrara A, Liu JY, et al. Missed appointments and poor glycemic control: an opportunity to identify high-risk diabetic patients. Med Care. 2004;42(2):110-5.

41. Griffin SJ. Lost to follow-up: The problem of defaulters from diabetes clinics. Diabet Med. 1998;15(Suppl 3):S14-24.

42. Lee RRS, Samsudin MI, Thirumoorthy T, Low LL, Kwan YH. Factors affecting follow-up non-attendance in patients with Type 2 diabetes mellitus and hypertension: a systematic review. Singapore Med J. 2019;60(5):216-23.

43. Sun C-A, Taylor K, Levin S, Renda SM, Han H-R. Factors associated with missed appointments by adults with type 2 diabetes mellitus: a systematic review. BMJ Open Diabetes Res Care. 2021;9(1):e001819.

44. Lin $\mathrm{H}, \mathrm{Wu} \mathrm{X}$. Intervention strategies for improving patient adherence to follow-up in the era of mobile information technology: a systematic review and meta-analysis. PLOS ONE. 2014;9(8):e104266.

45. Youssef A. Use of short message service reminders to improve attendance at an internal medicine outpatient clinic in Saudi Arabia: a randomized controlled trial. East Mediterr Health J. 2014;20(5):317-23.

Publisher's Note Springer Nature remains neutral with regard to jurisdictional claims in published maps and institutional affiliations. 